\section{Idosos no contexto da pandemia da COVID-19 no Brasil: efeitos nas condições de saúde, renda e trabalho}

\author{
Older adults in the context of the COVID-19 \\ pandemic in Brazil: effects on health, \\ income and work
}

\author{
Ancianos en el contexto de la pandemia de \\ COVID-19 en Brasil: efectos en las condiciones \\ de salud, renta y trabajo
}

\author{
Dalia Elena Romero 1 \\ Jéssica Muzy 1 \\ Giseli Nogueira Damacena 1 \\ Nathalia Andrade de Souza 1 \\ Wanessa da Silva de Almeida 1 \\ Celia Landmann Szwarcwald 1 \\ Deborah Carvalho Malta 2 \\ Marilisa Berti de Azevedo Barros 3 \\ Paulo Roberto Borges de Souza Júnior 1 \\ Luiz Otávio Azevedo 1 \\ Renata Gracie 1 \\ Maria de Fátima de Pina 1 \\ Margareth Guimarães Lima 3 \\ Ísis Eloah Machado 4 \\ Crizian Saar Gomes 5 \\ André Oliveira Werneck 6 \\ Danilo Rodrigues Pereira da Silva 7
}

doi: 10.1590/0102-311X00216620

\section{Resumo}

O presente estudo tem o objetivo de caracterizar a população idosa brasileira durante a pandemia de COVID-19, considerando suas condições de saúde, socioeconômicas, desigualdade de sexo, adesão ao distanciamento social e sentimento de tristeza ou depressão. Estudo transversal realizado com idosos brasileiros que participaram de um inquérito de saúde $(N=9.173)$, com método de amostragem "bola de neve virtual". Os dados foram coletados via web, por meio de questionário autopreenchido. Foram estimadas prevalências, intervalos de confiança e, para verificar a independência das estimativas, utilizou-se o teste qui-quadrado de Pearson. Durante a pandemia, houve diminuição da renda em quase metade dos domicílios dos idosos. O distanciamento social total foi adotado por 30,9\% (IC95\%: 27,8; 34,1) e 12,2\% (IC95\%: 10,1; 14,7) não aderiram. Idosos que não trabalhavam antes da pandemia aderiram em maior número às medidas de distanciamento social total. Grande parte apresentou comorbidades associadas ao maior risco de desenvolvimento da forma grave de COVID-19. Sentimentos de solidão, ansiedade e tristeza foram frequentes entre os idosos, especialmente entre as mulheres. A pandemia da COVID-19 aprofundou a desigualdade ao afetar os idosos mais vulneráveis. Estratégias para mitigar a solidão e o distanciamento social devem ser feitas levando-se em conta a vulnerabilidade social e a acentuada diferença entre homens e mulheres quanto à composição domiciliar e às condições socioeconômicas e de trabalho. Recomenda-se o desenvolvimento de pesquisas representativas da população idosa brasileira e que investiguem o impacto da pandemia neste grupo.

\section{Correspondência}

D. E. Romero

Instituto de Comunicação e Informação Científica e Tecnológica em Saúde, Fundação Oswaldo Cruz.

Av. Brasil 4365, sala 216, Rio de Janeiro, RJ 21040-360, Brasil. dalia.fiocruz@gmail.com

\footnotetext{
${ }_{1}$ Instituto de Comunicação e Informação Científica e Tecnológica em Saúde, Fundação Oswaldo Cruz, Rio de Janeiro, Brasil.

2 Escola de Enfermagem, Universidade Federal de Minas Gerais, Belo Horizonte, Brasil.

3 Faculdade de Ciências Médicas, Universidade Estadual de Campinas, Campinas, Brasil.

4 Escola de Medicina, Universidade Federal de Ouro Preto, Ouro Preto, Brasil.

5 Faculdade de Medicina, Universidade Federal de Minas Gerais, Belo Horizonte, Brasil.

6 Faculdade de Saúde Pública, Universidade de São Paulo, São Paulo, Brasil.

7 Centro de Ciências Biológicas e da Saúde, Universidade Federal de Sergipe, São Cristóvão, Brasil.
} 


\section{Introdução}

A pandemia da COVID-19 foi decretada pela Organização Mundial da Saúde em 11 de março de 2020 1. Desde as primeiras análises, em vários países mostrou-se que pessoas maiores de 60 anos são mais vulneráveis à doença 2. No Brasil, dados do Sistema de Informação de Vigilância Epidemiológica da Gripe (SIVEP-Gripe) apontam que até o dia 3 de junho de 2020 ocorreram 35.126 óbitos de pessoas idosas, o que corresponde a 71\% do total de óbitos por COVID-19, no período (Instituto de Comunicação e Informação Científica e Tecnológica em Saúde, Fundação Oswaldo Cruz. MonitoraCovid-19. https://bigdata-covid19.icict.fiocruz.br/, acessado em 13/Jun/2020). Segundo a Organização das Nações Unidas (ONU), na medida em que o vírus se espalha em países com debilitados sistemas de proteção social, a taxa de mortalidade para as pessoas idosas pode crescer ainda mais ${ }^{3}$. Além da grande ameaça à vida, a pandemia pode colocar pessoas idosas em maior risco de pobreza, perda de suporte social, trauma de estigma, discriminação e isolamento 4.

Nesse cenário, a perda do rendimento familiar durante a pandemia poderá agravar as desigualdades sociais e de saúde 5 . A influência da perda do trabalho sobre os distúrbios psicossociais foi, igualmente, documentada na literatura internacional 6 , podendo afetar a esperança de vida saudável.

A pandemia coincide com o envelhecimento populacional, considerado o principal evento demográfico do século XXI nos níveis mundial 7 e nacional 8. A Constituição brasileira, no seu Art. 230, dispõe que, além da família, a sociedade e o Estado têm o dever de amparar as pessoas idosas, "defendendo sua dignidade e bem-estar e garantindo-lhes o direito à vida". Além disso, o Brasil, como signatário do Plano Internacional de Envelhecimento de 2002 , tem o compromisso de reconhecer a vulnerabilidade dos idosos em situações de emergência humanitária, como é o caso de uma pandemia.

O sociólogo Norbert Elias 10, no seu livro Solidão dos Moribundos: Seguido de Envelhecer e Morrer, afirma que envelhecer está relacionado com distanciamento social, invisibilidade, luto e abandono. Essas questões preocupam ainda mais no contexto atual da inesperada pandemia da COVID-19.

O presente estudo tem o objetivo de caracterizar a população idosa brasileira durante a pandemia de COVID-19, considerando suas condições de saúde, socioeconômicas, desigualdade de sexo, adesão ao distanciamento social e sentimento de tristeza ou depressão.

\section{Métodos}

\section{Fonte de dados}

Trata-se de estudo descritivo com base nos dados da ConVid - Pesquisa de Comportamentos (ConVid) 11, inquérito de saúde de corte-transversal realizado, em âmbito nacional, pela Fundação Oswaldo Cruz, em parceria com a Universidade Federal de Minas Gerais e a Universidade Estadual de Campinas, no período de distanciamento social/quarentena consequente à pandemia. A coleta de dados foi realizada com a utilização de um questionário virtual, autopreenchido por meio de celular ou computador com acesso à Internet entre 24 de abril e 24 de maio de 2020. O projeto foi aprovado pela Comissão Nacional de Ética em Pesquisa em 19 de abril de 2020, parecer no 3.980.277.

A ConVid teve como objetivo descrever a aderência da população brasileira às medidas de restrição social, investigar as mudanças na situação de trabalho e rendimento, analisar dificuldades na realização das atividades de rotina, condições de saúde e descrever as mudanças de comportamentos adotados durante a pandemia de COVID-19. A ConVid utiliza questões validadas em inquéritos de saúde aplicados, previamente, no Brasil. Todas as respostas foram anônimas, sem qualquer tipo de identificação dos participantes, e armazenadas no servidor do Instituto de Comunicação e Informação Científica e Tecnológica em Saúde da Fundação Oswaldo Cruz (ICICT/Fiocruz).

Os critérios de inclusão para participação na pesquisa foram: ter 18 anos ou mais no momento do preenchimento e residir no território brasileiro durante a pandemia da COVID-19. Maiores detalhes da pesquisa ConVid estão no site da pesquisa (https://convid.fiocruz.br/) e em publicação recente sobre a metodologia 11 . 


\section{Amostra}

A amostragem foi realizada pelo método "bola de neve virtual", a partir do envio de convites para o questionário eletrônico, por redes sociais. Na primeira etapa, 15 pesquisadores do estudo selecionaram aproximadamente 200 outros pesquisadores de diferentes estados do Brasil. Cada pesquisador selecionou 20 pessoas da sua rede social, totalizando cerca de 500 pessoas (primeira onda), chamadas sementes. Para diversificar a amostra 12, as sementes convidaram pessoas das suas redes sociais, obedecendo a uma estratificação por sexo, faixa de idade e grau de escolaridade. Suas convidadas compuseram a segunda onda de recrutamento e enviaram novos convites, atingindo ao final uma amostra de 45.161 pessoas.

Para obter uma amostra representativa da população, foram realizadas ponderações por procedimentos de pós-estratificação 13, segundo: Unidade da Federação, capital, sexo, faixa etária, escolaridade e raça/cor com base nas estimativas da Pesquisa Nacional por Amostra de Domicílios de 2019 (PNAD 2019). Maiores detalhes sobre os procedimentos de pós-estratificação podem ser consultados em Szwarcwald et al. 11.

No presente estudo, foram analisadas as informações da população idosa (de 60 anos ou mais), totalizando 9.173 participantes, o que corresponde a 20,3\% da amostra total da pesquisa.

\section{Variáveis}

As caraterísticas demográficas consideradas foram: sexo e composição familiar, considerando-se as variáveis presença do cônjuge ou companheiro e situação de moradia (mora sozinho(a); mora com uma pessoa; mora com duas pessoas ou mais).

As variáveis sobre a situação laboral foram: trabalho antes da pandemia (sim; aposentado; não trabalhou por outra razão ou trabalhou sem remuneração) e vínculo empregatício (sim - que inclui servidores, trabalhadores com carteira assinada e donos de empresa; não - empregados sem carteira de trabalho e trabalhadores por conta própria). Durante a pandemia, foi investigado se o trabalho era atividade essencial (como assistência à saúde, segurança, transporte, serviço bancário etc.) e se houve mudança na situação laboral (não trabalhava antes e continuou sem trabalhar; continuou trabalhando normalmente; continuou trabalhando em casa; começou a trabalhar após a pandemia; teve férias remuneradas; perdeu o emprego; ficou sem trabalhar).

A renda per capita domiciliar em salários mínimos foi classificada como: menos de 1; de 1 a menos de 2; de 2 a menos de 4; 4 ou mais. O efeito na renda familiar foi obtido pela questão: "Como a pandemia da COVID-19 afetou a renda da família?" (aumentou ou foi mantida igual; diminuiu um pouco; diminuiu muito ou ficou sem renda). Também foi investigado se alguém do domicílio recebeu benefício financeiro do governo relacionado à pandemia de COVID-19.

A adesão ao distanciamento social na pandemia foi estimada pela pergunta: "Durante a pandemia do novo coronavírus, em que intensidade você fez (ou ainda está fazendo) distanciamento do contato com as pessoas?" (distanciamento total: fiquei rigorosamente em casa, saindo só por necessidades de atendimento à saúde; distanciamento intenso: fiquei em casa só saindo para compras em supermercado e farmácia; e não fez distanciamento ou pouco alterou sua rotina: não fiz nada, levei vida normal ou procurei tomar cuidados, ficar à distância das pessoas, reduzir um pouco o contato, não visitar idosos, mas continuei trabalhando e saindo).

As doenças crônicas não transmissíveis (DCNT) consideradas como risco para COVID-19 grave foram: diabetes, hipertensão, asma/enfisema/doença respiratória crônica ou outra doença do pulmão, doença do coração, e câncer 14 , obtidas com base na resposta positiva à pergunta: "Algum médico já lhe deu o diagnóstico de alguma destas doenças?”. O número de DCNT associadas ao risco para COVID-19 grave foi obtida baseando-se na contagem de DCNT de risco para COVID-19 declarada. A prevalência de fumantes foi obtida pela resposta positiva à pergunta: "Você é fumante?".

Os efeitos sobre o estado de saúde foram analisados por duas questões: "A pandemia provocou mudanças no estado de saúde?" (ficou igual; melhorou; piorou) e resposta positiva para: "Procurou atendimento de saúde durante a pandemia?”.

Sintomas associados à COVID-19 foram obtidos da resposta positiva a pelo menos um dos seguintes sintomas: febre, calafrio, dor de cabeça, tosse seca ou com catarro, fadiga, cansaço, dor de garganta, 
coriza, congestão nasal, náusea, vômito, diarreia ou dor nas articulações. Adicionalmente, utilizou-se a resposta à pergunta sobre a realização de testes para saber se estava infectado e seus resultados.

Os sentimentos de ansiedade ou nervosismo foram obtidos por autorreferência com base na seguinte pergunta: "No período da pandemia, com que frequência você se sentiu ansioso(a) ou nervoso(a)?” (nunca; poucas vezes; muitas vezes/sempre). Questões similares foram usadas para analisar os sentimentos de depressão ou tristeza, e de solidão pelo distanciamento de familiares e amigos, assim como em Barros et al. 15. Adicionalmente, foi investigada a resposta positiva à pergunta: "Algum familiar, amigo ou colega teve caso grave da COVID-19?"

$\mathrm{O}$ efeito da pandemia na renda dos idosos (aumentou ou foi mantida igual; diminuiu pouco; diminuiu muito ou ficou sem renda) foi relacionado às condições de trabalho, à renda per capita do domicílio e aos sentimentos frequentes de ansiedade ou nervosismo durante a pandemia.

\section{Análise}

As variáveis descritas foram analisadas segundo sexo e segundo intensidade da adesão ao distanciamento social durante a pandemia, por meio de sua distribuição percentual e respectivos intervalos de 95\% de confiança (IC95\%) das proporções estimadas. Para verificar a independência das estimativas, utilizou-se o teste qui-quadrado de Pearson.

As análises foram realizadas no pacote estatístico SPSS (https://www.ibm.com/), levando-se em consideração o peso amostral obtido para calibração da amostra.

\section{Resultados}

Os 9.173 indivíduos idosos que participaram da pesquisa ConVid, subdividiram-se em 3.969 homens e 5.204 mulheres.

A condição da moradia foi acentuadamente diferente entre homens e mulheres. Homens idosos vivem mais com cônjuge do que as idosas, 81,3\% (IC95\%: 76,3; 85,5) e 46\% (IC95\%: 42,0; 50,0), respectivamente. Já elas moram sozinhas $(23,8 \%$; IC95\%: 20,6; 27,3) mais frequentemente do que os homens (Tabela 1).

A atividade econômica é frequente entre os idosos. Metade deles trabalhou antes da pandemia (IC95\%: 47,0; 53,9), sendo 42,1\% (IC95\%: 37,4; 47,0) sem vínculo empregatício. A proporção de idosos que tinham vínculo era superior entre os homens $(62,6 \%$; IC95\%: 55,$5 ; 69,2)$ em relação às mulheres (53,1\%; IC95\%: 46,7; 59,3). Durante a pandemia, apenas 8,3\% (IC95\%: 6,4; 10,7) continuaram trabalhando normalmente e $21,2 \%$ (IC95\%: 18,4; 24,4$)$ trabalhando em casa. Um terço dos idosos $(33,9 \%$; IC95\%: 27,8; 40,6) trabalhou em atividades essenciais (Tabela 1).

A renda familiar foi menor que um salário mínimo para 31,9\% (IC95\%: 28,4; 35,6) dos idosos. Durante a pandemia, houve a diminuição da renda em quase metade dos domicílios dos idosos, onde 23,5\% (IC95\%: 20,6; 26,6) apresentaram pouca diminuição e 23,6\% (IC95\%: 20,7; 26,7) tiveram diminuição muito acentuada ou ficaram sem renda. Benefícios financeiros relacionados à pandemia da COVID-19 foram recebidos por 12\% (IC95\%: 9,4; 15,3) das famílias que moram com idosos (Tabela 1).

O distanciamento social total foi adotado por 30,9\% (IC95\%: 27,8; 34,1) dos idosos, 56,9\% (IC95\%: $53,5 ; 60,3)$ fizeram distanciamento social intenso e 12,2\% (IC95\%: 10,1; 14,7) não aderiram (ou aderiram pouco) às medidas de distanciamento. $O$ distanciamento total foi mais acentuado entre as mulheres $(39,1 \%$; IC95\%: 35,3; 42,9) do que entre os homens (20,2\%; IC95\%: 15,6; 25,8). Nota-se que apenas $7 \%$ (IC95\%: 5,4; 8,8) das mulheres fizeram pouco ou nenhum distanciamento (Tabela 2).

Idosos aposentados ou que já não trabalhavam antes da pandemia apresentaram maior adesão às medidas de distanciamento social total (40,4\%; IC95\%: 34,8; 46,3 e 41,7\%; IC95\%: 33,8; 50,0). Cerca de $10 \%$ dos idosos que continuaram trabalhando em home office ainda assim não aderiram ao distanciamento (IC95\%: 5,4; 17,4). Dos que exerceram alguma atividade essencial durante a pandemia, $44,2 \%$ (IC95\%: 33,9; 55,1) não aderiram ao distanciamento social. A renda não afetou significativamente o distanciamento social, sendo ele similar entre todas as faixas analisadas (Tabela 2).

As condições de saúde dos idosos durante a pandemia, segundo sexo, são mostradas na Tabela 3. A hipertensão é a DCNT com a maior prevalência entre as pessoas idosas (43,8\%; IC95\%: 38,5; 45,2). 
Tabela 1

Distribuição (\%) do arranjo familiar, situação da renda e do trabalho dos idosos segundo sexo, durante a pandemia de COVID-19. Brasil, 2020.

\begin{tabular}{|c|c|c|c|c|c|c|c|}
\hline \multirow[t]{2}{*}{ Situação socioeconômica e demográfica } & \multicolumn{2}{|c|}{$\begin{array}{l}\text { Masculino } \\
(\mathrm{n}=3.969)\end{array}$} & \multicolumn{2}{|c|}{$\begin{array}{l}\text { Feminino } \\
(n=5.204)\end{array}$} & \multicolumn{2}{|c|}{$\begin{array}{l}\text { Total } \\
(\mathrm{N}=9.173)\end{array}$} & \multirow[t]{2}{*}{ Valor de $p$} \\
\hline & $\%$ & IC95\% & $\%$ & IC95\% & $\%$ & IC95\% & \\
\hline Vive com cônjuge ou companheiro? & & & & & & & $<0,001$ \\
\hline $\operatorname{sim}$ & 81,3 & 76,$3 ; 85,5$ & 46 & 42,$0 ; 50,0$ & 61,3 & 57,$9 ; 64,6$ & \\
\hline Não & 18,7 & 14,$5 ; 23,7$ & 54 & 50,$0 ; 58,0$ & 38,7 & 35,$4 ; 42,1$ & \\
\hline Situação de moradia & & & & & & & $<0,001$ \\
\hline Mora sozinho(a) & 10,8 & 7,$8 ; 14,8$ & 23,8 & 20,$6 ; 27,3$ & 18,2 & 15,$8 ; 20,8$ & \\
\hline Mora com uma pessoa & 39,8 & 34,$4 ; 45,5$ & 43,3 & 39,$3 ; 47,3$ & 41,8 & 38,$5 ; 45,2$ & \\
\hline Mora com duas pessoas ou mais & 49,4 & 43,$5 ; 55,3$ & 32,9 & 29,$3 ; 36,8$ & 40,1 & 36,$6 ; 43,6$ & \\
\hline Trabalhava antes da pandemia? & & & & & & & $<0,001$ \\
\hline Sim & 59,3 & 53,$3 ; 65,1$ & 43,8 & 39,$8 ; 47,9$ & 50,5 & 47,$0 ; 53,9$ & \\
\hline Aposentado & 37,5 & 31,$8 ; 43,6$ & 39,7 & 35,$8 ; 43,7$ & 38,7 & 35,$4 ; 42,2$ & \\
\hline Não trabalhou por outra razão & 3,2 & 2,$0 ; 5,1$ & 16,6 & 13,$9 ; 19,5$ & 10,8 & 9,$1 ; 12,7$ & \\
\hline Se trabalhava, tinha vínculo empregatício? $(n=4.588)$ & & & & & & & 0,048 \\
\hline Sim & 62,6 & 55,$5 ; 69,2$ & 53,1 & 46,$7 ; 59,3$ & 57,9 & 53,$0 ; 62,6$ & \\
\hline Não & 37,4 & 30,$8 ; 44,5$ & 46,9 & 40,$7 ; 53,3$ & 42,1 & 37,$4 ; 47,0$ & \\
\hline Como a pandemia afetou a ocupação/trabalho? & & & & & & & $<0,001$ \\
\hline Não trabalhava antes e continuou sem trabalhar & 35,9 & 30,$5 ; 41,8$ & 49,6 & 45,$5 ; 53,8$ & 43,6 & 40,$3 ; 47$ & \\
\hline Continuou trabalhando normalmente & 11,3 & 7,$6 ; 16,4$ & 6 & 4,$6 ; 7,9$ & 8,3 & 6,$4 ; 10,7$ & \\
\hline Continuou trabalhando, mas em casa (home office) & 27,6 & 22,$6 ; 33,3$ & 16,2 & 13,$3 ; 19,6$ & 21,2 & 18,$4 ; 24,4$ & \\
\hline Começou a trabalhar após a pandemia & 0,2 & 0,$0 ; 0,8$ & 0,8 & 0,$4 ; 1,4$ & 0,5 & 0,$3 ; 0,9$ & \\
\hline Teve férias remuneradas & 3,1 & 1,$9 ; 5,2$ & 2,7 & 1,$6 ; 4,4$ & 2,9 & 2,$0 ; 4,1$ & \\
\hline Perdeu o emprego & 1,8 & 0,$6 ; 5,1$ & 0,6 & 0,$3 ; 1,2$ & 1,1 & 0,$5 ; 2,4$ & \\
\hline Ficou sem trabalhar & 20,1 & 15,$8 ; 25,1$ & 24,1 & 20,$2 ; 28,4$ & 22,3 & 19,$4 ; 25,6$ & \\
\hline $\begin{array}{l}\text { No trabalho durante a pandemia, exerceu alguma atividade } \\
\text { considerada essencial? }(n=2.645)\end{array}$ & & & & & & & 0,385 \\
\hline Sim & 31,5 & 23,$4 ; 41,0$ & 37,1 & 28,$8 ; 46,3$ & 33,9 & 27,$8 ; 40,6$ & \\
\hline Não & 68,5 & 59,$0 ; 76,6$ & 62,9 & 53,$7 ; 71,2$ & 66,1 & 59,$4 ; 72,2$ & \\
\hline Renda per capita domiciliar (salários mínimos) & & & & & & & 0,348 \\
\hline$<1$ & 29,3 & 23,$5 ; 35,8$ & 34,0 & $30,0-38,4$ & 31,9 & 28,$4 ; 35,6$ & \\
\hline $1<2$ & 28,2 & 22,$9 ; 34,2$ & 28,9 & 25,$1 ; 33,0$ & 28,6 & 25,$4 ; 32,0$ & \\
\hline $2<4$ & 25,3 & 20,$8 ; 30,4$ & 23,2 & 20,$2 ; 26,5$ & 24,2 & 21,$5 ; 27,0$ & \\
\hline$\geq 4$ & 17,2 & 14,$1 ; 20,9$ & 13,8 & 11,$8 ; 16,2$ & 15,3 & 13,$5 ; 17,4$ & \\
\hline Como a pandemia afetou a renda da família? & & & & & & & 0,431 \\
\hline Aumentou ou foi mantida igual & 53,6 & 47,$6 ; 59,4$ & 52,5 & 48,$4 ; 56,6$ & 53,0 & 49,$5 ; 56,4$ & \\
\hline Diminuiu um pouco & 23,8 & 18,$8 ; 29,6$ & 23,2 & 20,$1 ; 26,6$ & 23,5 & 20,$6 ; 26,6$ & \\
\hline Diminuiu muito ou ficou sem renda & 22,7 & 18,$4 ; 27,7$ & 24,3 & 20,$5 ; 28,4$ & 23,6 & 20,$7 ; 26,7$ & \\
\hline $\begin{array}{l}\text { Alguém do domicílio recebeu algum benefício do governo } \\
\text { relacionado à pandemia? }\end{array}$ & & & & & & & 0,843 \\
\hline Sim & 12,4 & 8,$1 ; 18,5$ & 11,8 & 8,$7 ; 15,7$ & 12,0 & 9,$4 ; 15,3$ & \\
\hline Não & 87,6 & 81,$5 ; 91,9$ & 88,2 & 84,$3 ; 91,3$ & 88,0 & 84,$7 ; 90,6$ & \\
\hline
\end{tabular}

IC95\%: intervalo de 95\% de confiança.

Fonte: ConVid - Pesquisa de Comportamentos. 
Tabela 2

Distribuição (\%) da situação socioeconômica, do domicílio e de trabalho dos idosos segundo a adesão ao distanciamento social durante a pandemia de COVID-19. Brasil, 2020

\begin{tabular}{|c|c|c|c|c|c|c|c|}
\hline \multirow[t]{2}{*}{$\begin{array}{l}\text { Situação socioeconômica e } \\
\text { demográfica }\end{array}$} & \multicolumn{2}{|c|}{ Distanciamento total } & \multicolumn{2}{|c|}{$\begin{array}{c}\text { Distanciamento } \\
\text { intenso (saiu para } \\
\text { mercado, compras etc.) }\end{array}$} & \multicolumn{2}{|c|}{$\begin{array}{l}\text { Não fez distanciamento ou } \\
\text { pouco alterou sua rotina }\end{array}$} & \multirow[t]{2}{*}{ Valor de $p$} \\
\hline & $\%$ & IC95\% & $\%$ & IC95\% & $\%$ & IC95\% & \\
\hline Total de idosos $(n=9.102)$ & 30,9 & 27,$8 ; 34,1$ & 56,9 & 53,$5 ; 60,3$ & 12,2 & 10,$1 ; 14,7$ & - \\
\hline Sexo & & & & & & & $<0,001$ \\
\hline Masculino & 20,2 & 15,$6 ; 25,8$ & 61,0 & 54,$7 ; 66,5$ & 19,0 & 14,$7 ; 24,2$ & \\
\hline Feminino & 39,1 & 35,$3 ; 42,9$ & 54,0 & 50,$0 ; 57,9$ & 7,0 & 5,$4 ; 8,8$ & \\
\hline Vive com cônjuge ou companheiro? & & & & & & & 0,001 \\
\hline Sim & 26,8 & 23,$0 ; 30,9$ & 58,7 & 54,$3 ; 63,1$ & 14,5 & 11,$6 ; 17,9$ & \\
\hline Não & 37,7 & 32,$8 ; 42,8$ & 54,2 & 49,$0 ; 59,4$ & 8,1 & 5,$4 ; 12,1$ & \\
\hline Situação de moradia & & & & & & & 0,087 \\
\hline Mora sozinho(a) & 35,1 & 28,$5 ; 42,4$ & 58,4 & 51,$1 ; 65,4$ & 6,5 & 3,$8 ; 10,7$ & \\
\hline Mora com uma pessoa & 29,6 & 25,$8 ; 33,8$ & 58,3 & 53,$5 ; 63,0$ & 12,1 & 8,$8 ; 16,2$ & \\
\hline Mora com duas pessoas ou mais & 30,2 & 24,$8 ; 36,2$ & 54,8 & 48,$9 ; 60,7$ & 15,0 & 11,$4 ; 19,6$ & \\
\hline Trabalhava antes da pandemia? & & & & & & & $<0,001$ \\
\hline Sim & 20,8 & 17,$6 ; 24,3$ & 58,8 & 54,$0 ; 63,3$ & 20,5 & 16,$6 ; 25,0$ & \\
\hline Aposentado & 40,4 & 34,$8 ; 46,3$ & 56,3 & 50,$4 ; 62,0$ & 3,3 & 2,$1 ; 5,4$ & \\
\hline Não trabalhou por outra razão & 41,7 & 33,$8 ; 50,0$ & 54,3 & 46,$0 ; 62,4$ & 4,0 & 2,$3 ; 6,9$ & \\
\hline $\begin{array}{l}\text { Como a pandemia afetou a ocupação/ } \\
\text { trabalho? }\end{array}$ & & & & & & & $<0,001$ \\
\hline $\begin{array}{l}\text { Não trabalhava antes e continuou } \\
\text { sem trabalhar }\end{array}$ & 37,4 & 33,$1 ; 41,8$ & 56,1 & 51,$4 ; 60,7$ & 6,5 & 4,$3 ; 9,9$ & \\
\hline $\begin{array}{l}\text { Continuou trabalhando } \\
\text { normalmente }\end{array}$ & 15,2 & 5,$0 ; 37,8$ & 18,3 & 12,$2 ; 26,5$ & 66,6 & 51,$1 ; 79,2$ & \\
\hline $\begin{array}{l}\text { Continuou trabalhando, mas em } \\
\text { casa (home office) }\end{array}$ & 26,3 & 20,$5 ; 33,0$ & 63,8 & 56,$1 ; 70,9$ & 9,8 & 5,$4 ; 17,4$ & \\
\hline $\begin{array}{l}\text { Começou a trabalhar após a } \\
\text { pandemia }\end{array}$ & 19,0 & 5,$2 ; 50,1$ & 39,3 & 17,$4 ; 66,6$ & 41,7 & 17,$7 ; 70,3$ & \\
\hline Teve férias remuneradas & 18,4 & 8,$7 ; 34,8$ & 74,5 & 57,$6 ; 86,3$ & 7,1 & 2,$4 ; 19,4$ & \\
\hline Perdeu o emprego & 11,3 & 3,$1 ; 33,5$ & 82,7 & 57,$8 ; 94,4$ & 6,0 & 1,$3 ; 24,0$ & \\
\hline Ficou sem trabalhar & 29,4 & 22,$4 ; 37,5$ & 64,1 & 56,$0 ; 71,5$ & 6,5 & 3,$9 ; 10,7$ & \\
\hline $\begin{array}{l}\text { No trabalho durante a pandemia, } \\
\text { exerceu alguma atividade considerada } \\
\text { essencial? }\end{array}$ & & & & & & & $<0,001$ \\
\hline Sim & 12,2 & 7,$6 ; 19,2$ & 43,5 & 33,$4 ; 54,2$ & 44,2 & 33,$9 ; 55,1$ & \\
\hline Não & 28,0 & 20,$5 ; 36,9$ & 54,1 & 45,$4 ; 62,5$ & 17,9 & 12,$1 ; 25,8$ & \\
\hline $\begin{array}{l}\text { Renda per capita domiciliar (salários } \\
\text { mínimos) }\end{array}$ & & & & & & & 0,159 \\
\hline$<1$ & 33,5 & 27,$0 ; 40,8$ & 52,3 & 45,$2 ; 59,4$ & 14,1 & 10,$1 ; 19,4$ & \\
\hline $1<2$ & 24,1 & 19,$2 ; 29,9$ & 63,8 & 56,$9 ; 70,1$ & 12,1 & 7,$8 ; 18,3$ & \\
\hline $2<4$ & 29,1 & 23,$9 ; 34,9$ & 59,1 & 52,$9 ; 65,1$ & 11,8 & 8,$0 ; 16,9$ & \\
\hline$\geq 4$ & 35,6 & 29,$9 ; 41,7$ & 53,8 & 47,$4 ; 60,0$ & 10,7 & 6,$8 ; 16,5$ & \\
\hline $\begin{array}{l}\text { Como a pandemia afetou a renda da } \\
\text { família? }\end{array}$ & & & & & & & 0,218 \\
\hline Aumentou ou foi mantida igual & 32,7 & 28,$8 ; 36,9$ & 56,7 & 52,$2 ; 61,1$ & 10,6 & 8,$0 ; 13,9$ & \\
\hline Diminuiu pouco & 33,3 & 26,$3 ; 41,2$ & 53,2 & 45,$6 ; 60,6$ & 13,5 & 8,$6 ; 20,5$ & \\
\hline Diminuiu muito ou ficou sem renda & 24,0 & 18,$4 ; 30,7$ & 61,8 & 54,$7 ; 63,9$ & 14,2 & 10,$4 ; 19,3$ & \\
\hline
\end{tabular}

IC95\%: intervalo de 95\% de confiança.

Fonte: ConVid - Pesquisa de Comportamentos. 
Tabela 3

Distribuição (\%) das doenças crônicas não transmissíveis (DCNT) de risco para a COVID-19, comorbidades, estado de saúde e sintomas de COVID-19 e sentimentos de tristeza, ansiedade e solidão segundo sexo. Brasil, 2020.

\begin{tabular}{|c|c|c|c|c|c|c|c|}
\hline \multirow[t]{2}{*}{ Situação de saúde } & \multicolumn{2}{|c|}{ Masculino } & \multicolumn{2}{|c|}{ Feminino } & \multicolumn{2}{|c|}{ Total } & \multirow[t]{2}{*}{ Valor de $p$} \\
\hline & $\%$ & IC95\% & $\%$ & IC95\% & $\%$ & IC95\% & \\
\hline \multicolumn{8}{|l|}{ Prevalência das DCNT consideradas fatores de risco para a } \\
\hline \multicolumn{8}{|l|}{ COVID-19 } \\
\hline Diabetes & 18,7 & 14,$6 ; 23,5$ & 15,6 & 12,$6 ; 19,1$ & 16,9 & 14,$4 ; 19,7$ & 0,267 \\
\hline Hipertensão & 43,8 & 38,$1 ; 49,7$ & 40,3 & 36,$5 ; 44,3$ & 41,8 & 38,$5 ; 45,2$ & 0,331 \\
\hline $\begin{array}{l}\text { Asma/Enfisema/Doença respiratória crônica ou outra doença } \\
\text { do pulmão }\end{array}$ & 9,3 & 5,$7 ; 14,9$ & 9,6 & 7,$7 ; 12,0$ & 9,5 & 7,$4 ; 12,0$ & 0,899 \\
\hline Doença do coração & 15,6 & 11,$5 ; 20,9$ & 7,8 & 5,$4 ; 11,2$ & 11,2 & 8,$8 ; 14,1$ & 0,004 \\
\hline Câncer & 6,4 & 4,$1 ; 9,8$ & 7,0 & 5,$5 ; 8,9$ & 6,7 & 5,$3 ; 8,4$ & 0,711 \\
\hline \multicolumn{8}{|l|}{ Fatores de risco para COVID-19 } \\
\hline Tabagista & 12,0 & 8,$8 ; 16,1$ & 13,3 & 10,$9 ; 16,0$ & 12,7 & 10,$7 ; 15,0$ & 0,578 \\
\hline Pelo menos uma DCNT de risco COVID-19 & 60,2 & 54,$3 ; 65,8$ & 57,5 & 53,$5 ; 61,3$ & 58,6 & 55,$3 ; 61,9$ & 0,442 \\
\hline Pelo menos uma DCNT de risco COVID-19 ou fumante & 64,2 & 58,$4 ; 69,6$ & 64,1 & 60,$2 ; 67,7$ & 64,1 & 60,$8 ; 67,3$ & 0,966 \\
\hline Número de DCNT associadas ao risco para a COVID-19 & & & & & & & 0,047 \\
\hline Nenhuma & 39,8 & 34,$2 ; 45,7$ & 42,5 & 38,$7 ; 46,5$ & 41,4 & 38,$1 ; 44,7$ & \\
\hline Uma & 33,9 & 28,$5 ; 39,7$ & 38,4 & 34,$4 ; 42,6$ & 36,5 & 33,$2 ; 39,9$ & \\
\hline Duas & 19,1 & 15,$0 ; 24,1$ & 15,5 & 12,$9 ; 18,5$ & 17,1 & 14,$7 ; 19,8$ & \\
\hline Três & 7,1 & 4,$3 ; 11,7$ & 3,5 & 2,$6 ; 4,8$ & 5,1 & 3,$6 ; 7,1$ & \\
\hline A pandemia provocou mudanças no estado de saúde? & & & & & & & 0,062 \\
\hline Ficou igual & 82,2 & 75,$6 ; 78,5$ & 82,2 & 77,$4 ; 86,2$ & 75,6 & 72,$0 ; 78,9$ & \\
\hline Melhorou & 1,6 & 2,$5 ; 2,1$ & 1,6 & 0,$7 ; 3,3$ & 2,5 & 1,$6 ; 3,9$ & \\
\hline Piorou & 16,2 & 21,$9 ; 19,4$ & 16,2 & 12,$3 ; 21,0$ & 21,9 & 18,$7 ; 25,4$ & \\
\hline Procurou atendimento de saúde durante a pandemia & 15,7 & 12,$6 ; 19,4$ & 19,5 & 16,$6 ; 22,7$ & 17,9 & 15,$7 ; 20,3$ & 0,111 \\
\hline Teve algum sintoma associado à COVID-19 & 12,9 & 9,$6 ; 17,2$ & 18,4 & 15,$3 ; 22,0$ & 16,1 & 13,$7 ; 18,8$ & 0,040 \\
\hline Fez o teste para saber se estava infectado & 2,4 & 0,$8 ; 7,2$ & 2,0 & 1,$1 ; 3,9$ & 2,2 & 1,$2 ; 3,9$ & 0,798 \\
\hline Resultado do teste $(n=79)$ & & & & & & & 0,011 \\
\hline Positivo & 23,2 & 6,$1 ; 58,2$ & 2,4 & 1,$1 ; 5,2$ & 6,0 & 2,$8 ; 12,4$ & \\
\hline Negativo & 27,0 & 7,$7 ; 62,1$ & 83,6 & 60,$2 ; 94,5$ & 73,9 & 49,$6 ; 89,1$ & \\
\hline Ainda não recebeu resultado & 49,9 & 11,$6 ; 88,3$ & 14,0 & 4,$1 ; 38,5$ & 20,1 & 6,$9 ; 46,2$ & \\
\hline $\begin{array}{l}\text { No período da pandemia, se sentiu isolado(a) de familiares ou } \\
\text { amigos? }\end{array}$ & & & & & & & $<0,001$ \\
\hline Nunca & 21,1 & 16,$6 ; 26,6$ & 15,5 & 12,$5 ; 19,1$ & 18,0 & 15,$3 ; 21,0$ & \\
\hline Poucas vezes & 37,8 & 32,$2 ; 43,8$ & 26,6 & 23,$7 ; 29,8$ & 31,5 & 28,$5 ; 34,7$ & \\
\hline Muitas vezes/Sempre & 41,0 & 35,$4 ; 46,9$ & 57,8 & 53,$9 ; 61,7$ & 50,6 & 47,$1 ; 54,0$ & \\
\hline No período da pandemia, se sentiu ansioso(a) ou nervoso(a)? & & & & & & & $<0,001$ \\
\hline Nunca & 29,3 & 24,$2 ; 34,9$ & 18,5 & 15,$6 ; 21,7$ & 23,1 & 20,$4 ; 26,2$ & \\
\hline Poucas vezes & 47,5 & 41,$6 ; 53,5$ & 43,4 & 39,$5 ; 47,4$ & 45,2 & 41,$8 ; 48,6$ & \\
\hline Muitas vezes/Sempre & 23,2 & 18,$8 ; 28,4$ & 38,1 & 34,$2 ; 42,2$ & 31,7 & 28,$6 ; 34,9$ & \\
\hline No período da pandemia, se sentiu triste ou deprimido? & & & & & & & $<0,001$ \\
\hline Nunca & 38,8 & 33,$2 ; 44,8$ & 20,8 & 17,$5 ; 24,5$ & 28,5 & 25,$4 ; 31,9$ & \\
\hline Poucas vezes & 43,7 & 37,$9 ; 49,7$ & 44,1 & 40,$2 ; 48,1$ & 43,9 & 40,$6 ; 47,4$ & \\
\hline Muitas vezes/Sempre & 17,5 & 13,$7 ; 22,1$ & 35,1 & 31,$3 ; 39,1$ & 27,5 & 24,$6 ; 30,6$ & \\
\hline Algum familiar, amigo ou colega teve caso grave da COVID-19? & 10,9 & 8,$2 ; 14,4$ & 15,6 & 12,$6 ; 19,2$ & 13,6 & 11,$4 ; 16,1$ & 0,044 \\
\hline
\end{tabular}

IC95\%: intervalo de 95\% de confiança.

Fonte: ConVid - Pesquisa de Comportamentos. 
Mais de 58\% (IC95\%: 55,3; 61,9) dos idosos têm pelo menos uma DCNT de risco para COVID-19 grave. Ao acrescentar o tabagismo como outro fator de risco para a severidade da COVID-19, observa-se que 64,1\% (IC95\%: 60,8; 67,3) da população idosa faz parte do grupo com acentuado risco, seja por ter pelo menos uma DCNT ou por ser fumante ativo. Homens apresentam mais comorbidades associadas ao risco para COVID-19 grave em relação às mulheres, 7,1\% deles registram três doenças ou mais (IC95\%: 4,3; 11,7) (Tabela 3).

A piora do estado de saúde durante a pandemia foi relatada por 21,9\% (IC95\%: 18,7; 25,4) dos idosos. A procura por um médico, dentista ou outro profissional de saúde foi realizada por 17,9\% (IC95\%: 15,7; 20,3) (Tabela 3).

Sintomas associados à COVID-19 foram relatados por 16,1\% (IC95\%: 13,7; 18,8) dos idosos que responderam à pesquisa, sendo 18,4\% (IC95\%: 15,3; 22,0) entre as mulheres e 12,9\% (IC95\%: 9,6; 17,2) entre os homens. Apenas 2,2\% (IC95\%: 1,2; 3,9) dos idosos fizeram o teste da COVID-19 (Tabela 3).

Sentimento frequente de solidão pelo distanciamento dos amigos e familiares na pandemia foi relatado por metade dos idosos (IC95\%: 47,1; 54,0), sendo que este sentimento é mais frequente na população idosa feminina, 57,8\% (IC95\%: 53,9; 61,7), em relação à masculina (41\%; IC95\%: 35,4; 46,9). Ansiedade ou nervosismo na maioria do tempo durante o período da pandemia foi relatado por $1 / 3$ da população idosa (31,7\%; IC95\%: 28,6; 34,9), sendo maior também entre as mulheres (38,1\%; IC95\%: $34,2 ; 42,2)$ do que entre os homens (23,2; IC95\%: 18,8; 28,4). O sentimento recorrente de tristeza e depressão $(27,5 \%$; IC95\%: 24,6; 30,6) também foi mais marcante na população feminina $(35,1 \%$; IC95\%: 31,3; 39,1) em comparação aos homens (17,5\%; IC95\%: 13,7; 22,1). Casos graves da COVID-19 em familiares, amigos próximos ou colegas de trabalho foram relatados por 13,6\% (IC95\%: 11,4; 16,1) dos idosos (Tabela 3).

A desigualdade e a intensidade do impacto da pandemia na renda dos idosos podem ser observadas na Tabela 4. No Brasil, 23,7\% (IC95\%: 20,8; 26,8) diminuíram muito ou perderam a renda no período pesquisado. Esse percentual é mais elevando entre os idosos que trabalharam antes da pandemia, 36,4\% (IC95\%: 31,8; 41,3). Observa-se que 52,4\% (IC95\%: 46,1; 58,7) dos idosos que tinham vínculo mantiveram sua renda, e apenas 20,3\% (IC95\%: 15,1; 26,7) dos que estavam sem vínculo não foram negativamente afetados. Grande parte dos idosos que não tinham vínculo empregatício pioraram muito a sua renda (55,3\%; IC95\%: 47,8; 62,5).

Foi observada uma parcela de idosos com perda significativa da renda mesmo entre os que continuaram trabalhando normalmente $(19,4 \%$; IC95\%: 13,$0 ; 27,9)$ ou em regime de home office $(23,0 \%$; IC95\%: 16,8; 30,7). A diminuição da renda afetou mais os idosos com renda per capita domiciliar menor que um salário mínimo (36,2\%; IC95\%: 29,5; 43,6).

O número de moradores no domicílio não implicou mudanças significativas na sensação de tristeza. A tristeza ou depressão recorrente foi mais expressiva em domicílios com menor renda (32,3\%; IC95\%: 26,2; 39,0) em relação às demais faixas. Igualmente, durante a pandemia, quanto maior a diminuição da renda tanto maior a proporção de idosos que estiveram tristes sempre ou muitas vezes, chegando a 38,5\% (IC95\%: 31,7; 45,7). Em geral, os idosos que conheciam algum familiar, amigo ou colega que teve caso grave ou falecimento por COVID-19 sentiram-se tristes mais frequentemente (37,1\%; IC95\%: 28,4; 46,7) (Tabela 5).

O sentimento de isolamento de familiares e amigos esteve relacionado à tristeza e depressão na população idosa, visto que 66,3\% (IC95\%: 51,1; 74,4) dos que nunca se sentiram isolados também não ficaram tristes ou deprimidos. Entre os que nunca se sentiram isolados do seu ciclo social, por exemplo, apenas 3,5\% (IC95\%: 1,8; 6,9) sentiram-se tristes sempre ou muitas vezes. Quando a tristeza é analisada em relação ao grau de distanciamento social adotado, idosos que aderiram ao distanciamento total sentiram-se tristes com mais frequência (30,5\%; IC95\%: 25,9; 35,6). 


\section{Tabela 4}

Distribuição (\%) das condições de trabalho e renda per capita domiciliar durante a pandemia de COVID-19, segundo o impacto na renda dos idosos. Brasil, 2020.

\begin{tabular}{|c|c|c|c|c|c|c|c|}
\hline \multirow[t]{3}{*}{ Situação socioeconômica } & \multicolumn{6}{|c|}{ Mudança da renda durante a pandemia } & \multirow[t]{3}{*}{ Valor de $p$} \\
\hline & \multicolumn{2}{|c|}{$\begin{array}{l}\text { Aumentou ou foi } \\
\text { mantida igual }\end{array}$} & \multicolumn{2}{|c|}{ Diminuiu pouco } & \multicolumn{2}{|c|}{$\begin{array}{l}\text { Diminuiu muito ou } \\
\text { ficou sem renda }\end{array}$} & \\
\hline & $\%$ & $\mathrm{IC} 95 \%$ & $\%$ & IC95\% & $\%$ & IC95\% & \\
\hline Total de idosos $(n=9.173)$ & 52,9 & 49,$4 ; 56,3$ & 23,4 & 20,$5 ; 26,6$ & 23,7 & 20,$8 ; 26,8$ & - \\
\hline Trabalhava antes da pandemia? & & & & & & & $<0,001$ \\
\hline Sim & 38,9 & 34,$3 ; 43,7$ & 24,7 & 20,$9 ; 29,0$ & 36,4 & 31,$8 ; 41,3$ & \\
\hline Aposentado & 70,0 & 63,$9 ; 75,5$ & 21,6 & 16,$7 ; 27,4$ & 8,4 & 5,$5 ; 12,6$ & \\
\hline Não trabalhou por outra razão & 57,3 & 48,$8 ; 65,3$ & 23,9 & 17,$7 ; 31,5$ & 18,8 & 13,$1 ; 26,3$ & \\
\hline Se trabalhava, tinha vínculo empregatício? $(\mathrm{n}=4.588)$ & & & & & & & $<0,001$ \\
\hline Sim & 52,4 & 46,$1 ; 58,7$ & 24,9 & 19,$8 ; 30,7$ & 22,7 & 17,$7 ; 28,6$ & \\
\hline Não & 20,3 & 15,$1 ; 26,7$ & 24,5 & 18,$9 ; 31,1$ & 55,3 & 47,$8 ; 62,5$ & \\
\hline Como a pandemia afetou a ocupação/trabalho? & & & & & & & $<0,001$ \\
\hline Não trabalhava antes e continuei sem trabalhar & 74,6 & 70,$2 ; 78,5$ & 18,0 & 14,$4 ; 22,3$ & 7,4 & 5,$6 ; 9,6$ & \\
\hline Continuei trabalhando & 44,3 & 31,$9 ; 57,5$ & 36,3 & 22,$9 ; 52,1$ & 19,4 & 13,$0 ; 27,9$ & \\
\hline Continuei trabalhando, mas em casa (home office) & 46,7 & 39,$0 ; 54,6$ & 30,3 & 23,$3 ; 38,3$ & 23,0 & 16,$8 ; 30,7$ & \\
\hline Comecei a trabalhar após a pandemia & 55,9 & 28,$8 ; 80,0$ & 14,7 & 3,$1 ; 48,1$ & 29,3 & 11,$0 ; 58,1$ & \\
\hline Tive férias remuneradas & 30,3 & 17,$4 ; 47,3$ & 36,4 & 20,$5 ; 56,1$ & 33,3 & 18,$7 ; 51,9$ & \\
\hline Perdi o emprego & 0,2 & 0,$0 ; 1,1$ & 9,6 & 3,$0 ; 26,4$ & 90,2 & 73,$4 ; 96,8$ & \\
\hline Fiquei sem trabalhar & 25,3 & 18,$5 ; 33,7$ & 22,1 & 17,$3 ; 27,8$ & 52,6 & 44,$6 ; 60,5$ & \\
\hline $\begin{array}{l}\text { No trabalho durante a pandemia, exerceu alguma } \\
\text { atividade considerada essencial? }\end{array}$ & & & & & & & 0,625 \\
\hline Sim & 43,0 & 32,$4 ; 54,4$ & 30,7 & 21,$4 ; 41,9$ & 26,2 & 16,$6 ; 38,8$ & \\
\hline Não & 47,2 & 39,$0 ; 55,7$ & 32,4 & 24,$1 ; 42,0$ & 20,3 & 15,$2 ; 26,7$ & \\
\hline Renda per capita domiciliar (salários mínimos) & & & & & & & $<0,001$ \\
\hline$<1$ & 38,5 & 31,$8 ; 45,5$ & 25,3 & 19,$2 ; 32,5$ & 36,2 & 29,$5 ; 43,6$ & \\
\hline $1<2$ & 56,5 & 49,$5 ; 63,2$ & 26,0 & 20,$3 ; 32,8$ & 17,5 & 13,$6 ; 22,3$ & \\
\hline $2<4$ & 65,2 & 59,$2 ; 70,7$ & 17,0 & 13,$1 ; 21,8$ & 17,8 & 13,$6 ; 23,0$ & \\
\hline$\geq 4$ & 61,4 & 55,$0 ; 67,5$ & 21,7 & 16,$9 ; 27,6$ & 16,9 & 12,$4 ; 22,5$ & \\
\hline
\end{tabular}

IC95\%: intervalo de 95\% de confiança.

Fonte: ConVid - Pesquisa de Comportamentos.

\section{Discussão}

Neste artigo, apresentam-se evidências do alto e desigual impacto da pandemia da COVID-19 na saúde, renda e cuidados dos idosos brasileiros. Esses efeitos são menos visíveis do que as altas taxas de letalidade e mortalidade 16 , mas representam sérias consequências para os idosos e suas famílias. Também foi mostrada acentuada diferença entre homens e mulheres idosos quanto à composição domiciliar, condições socioeconômicas, renda familiar e inserção no mercado de trabalho. Isso reforça que a desigualdade de gênero sempre deve ser considerada nos estudos e ações sobre o envelhecimento ${ }^{17}$. No período da pandemia da COVID-19, a composição domiciliar dos idosos representa risco independentemente da conformação. Idosos morando sozinhos podem precisar de ajuda para adquirir alimentos, suporte afetivo, econômico, cuidados à saúde e outros, e idosos que moram com outras pessoas correm risco de serem contagiados por integrantes do lar que mantenham contato com o exterior 18. Morar com outras pessoas é mais presente entre a população masculina, e morar sozinho é muito mais frequente entre a população feminina. Resultados similares foram encontrados em pesquisas anteriores 19,20 . 
Tabela 5

Distribuição (\%) da situação socioeconômica, do domicílio, do trabalho, da saúde e distanciamento dos idosos segundo a autopercepção de tristeza e depressão durante a pandemia de COVID-19. Brasil, 2020.

\begin{tabular}{|c|c|c|c|c|c|c|c|}
\hline \multirow[t]{3}{*}{ Situação socioeconômica, demográfica e de saúde } & \multicolumn{6}{|c|}{ No período da pandemia, se sentiu triste ou deprimido? } & \multirow[t]{3}{*}{ Valor de $p$} \\
\hline & \multicolumn{2}{|c|}{ Nunca } & \multicolumn{2}{|c|}{ Poucas vezes } & \multicolumn{2}{|c|}{ Muitas vezes/Sempre } & \\
\hline & $\%$ & IC95\% & $\%$ & IC95\% & $\%$ & IC95\% & \\
\hline Total de idosos $(n=9.133)$ & 28,5 & 25,$4 ; 31,9$ & 43,9 & 40,$6 ; 47,4$ & 27,5 & 24,$6 ; 30,6$ & - \\
\hline Sexo & & & & & & & $<0,001$ \\
\hline Masculino & 38,8 & 33,$2 ; 44,8$ & 43,7 & 37,$9 ; 49,7$ & 17,5 & 13,$7 ; 22,1$ & \\
\hline Feminino & 20,8 & 17,$5 ; 24,5$ & 44,1 & 40,$2 ; 48,1$ & 35,1 & 31,$3 ; 39,1$ & \\
\hline Situação de moradia & & & & & & & 0,101 \\
\hline Mora sozinho(a) & 25,9 & 12,$9 ; 45,1$ & 46,8 & 27,$8 ; 66,8$ & 27,3 & 14,$4 ; 45,5$ & \\
\hline Mora com uma pessoa & 28,7 & 23,$9 ; 34,0$ & 43,0 & 37,$9 ; 48,3$ & 28,3 & 23,$8 ; 33,3$ & \\
\hline Mora com duas pessoas ou mais & 28,4 & 24,$3 ; 32,9$ & 44,6 & 40,$1 ; 49,2$ & 26,9 & 23,$3 ; 30,9$ & \\
\hline Renda per capita domiciliar (salários mínimos) & & & & & & & 0,032 \\
\hline$<1$ & 23,3 & 17,$8 ; 30,0$ & 44,4 & 37,$2 ; 51,8$ & 32,3 & 26,$2 ; 39,0$ & \\
\hline $1<2$ & 33,9 & 27,$3 ; 41,3$ & 37,3 & 31,$1 ; 43,9$ & 28,7 & 22,$6 ; 35,7$ & \\
\hline $2<4$ & 27,7 & 22,$3 ; 33,8$ & 47,9 & 41,$7 ; 54,1$ & 24,4 & 19,$7 ; 29,8$ & \\
\hline$\geq 4$ & 28,4 & 23,$2 ; 34,1$ & 50,0 & 43,$7 ; 56,2$ & 21,7 & 17,$1 ; 27,1$ & \\
\hline Como a pandemia afetou a renda da família? & & & & & & & $<0,001$ \\
\hline Aumentou ou foi mantida igual & 33,7 & 29,$3 ; 38,4$ & 42,3 & 37,$9 ; 46,7$ & 24,0 & 20,$5 ; 28,0$ & \\
\hline Diminuiu pouco & 20,8 & 16,$2 ; 26,4$ & 54,9 & 47,$7 ; 62,0$ & 24,2 & 18,$7 ; 30,8$ & \\
\hline Diminuiu muito ou ficou sem renda & 24,9 & 18,$3 ; 33,0$ & 36,6 & 30,$1 ; 43,7$ & 38,5 & 31,$7 ; 45,7$ & \\
\hline $\begin{array}{l}\text { Algum familiar, amigo ou colega teve caso grave da } \\
\text { COVID-19? }\end{array}$ & & & & & & & 0,007 \\
\hline Sim & 17,4 & 11,$4 ; 25,6$ & 45,5 & 36,$7 ; 54,7$ & 37,1 & 28,$4 ; 46,7$ & \\
\hline Não & 30,4 & 26,$9 ; 34,1$ & 43,7 & 40,$0 ; 47,4$ & 26,0 & 23,$0 ; 29,2$ & \\
\hline $\begin{array}{l}\text { No período da pandemia, se sentiu isolado(a) de } \\
\text { familiares ou amigos? }\end{array}$ & & & & & & & $<0,001$ \\
\hline Nunca & 66,3 & 57,$1 ; 74,4$ & 30,2 & 22,$3 ; 39,5$ & 3,5 & 1,$8 ; 6,9$ & \\
\hline Poucas vezes & 33,7 & 28,$4 ; 39,4$ & 56,4 & 50,$6 ; 62,0$ & 9,9 & 7,$4 ; 13,3$ & \\
\hline Muitas vezes/sempre & 11,9 & 8,$5 ; 16,3$ & 41,0 & 36,$5 ; 45,6$ & 47,1 & 42,$4 ; 51,9$ & \\
\hline Durante a pandemia, fez distanciamento social? & & & & & & & 0,006 \\
\hline Sim, distanciamento total & 22,1 & 17,$5 ; 27,6$ & 47,4 & 41,$4 ; 53,5$ & 30,5 & 25,$9 ; 35,6$ & \\
\hline $\begin{array}{l}\text { Sim, distanciamento intenso saiu para mercado, } \\
\text { compras etc. }\end{array}$ & 29,9 & 25,$6 ; 34,6$ & 45,2 & 40,$8 ; 49,7$ & 24,9 & 21,$3 ; 28,8$ & \\
\hline Não fez distanciamento ou pouco alterou sua rotina & 39,7 & 30,$2 ; 49,9$ & 31,2 & 23,$2 ; 40,5$ & 29,1 & 20,$0 ; 40,4$ & \\
\hline
\end{tabular}

IC95\%: intervalo de 95\% de confiança.

Fonte: ConVid - Pesquisa de Comportamentos.

Morar sozinho e envelhecer são considerados os fenômenos demográficos mais relevantes das últimas décadas 21. A pandemia atual e as recomendações de distanciamento que vieram a seguir aumentaram a consciência pública sobre os impactos psicológicos das medidas de distanciamento social e da solidão que muitas pessoas estão experimentando 22,23 . Na vida cotidiana de muitos idosos esse sentimento é comum, entretanto, silenciado pela sociedade 24 .

A solidão, importante preditor de mortalidade e de fatores de risco clínicos na velhice, como o declínio da capacidade funcional, deveria ser tratada como grave fator de risco e problema de saúde 25,26. Sabe-se que a solidão no envelhecimento pode antecipar a morte e precipitar agravos, já que está associada a riscos de desenvolver doenças coronárias e derrame, independentemente dos fatores de risco tradicionais para as doenças cardiovasculares 26 . 
Idosos podem estar isolados socialmente sem relatar sentimento de solidão e podem estar sozinhos mesmo sem isolamento social, mas ambas as condições podem causar danos à sua saúde física e mental 2,22. Assim, distanciamento social não deve ser confundido com solidão 27 . O sentimento de tristeza é o que mais contribui para a solidão 28 e se sentir sozinho não é apenas a ausência de acompanhante no lar. Neste trabalho, verificou-se que é frequente idosos brasileiros se sentirem muitas vezes ou sempre sozinhos. Esse sentimento está associado, geralmente, com o problema estrutural sobre luto, abandono social e estigma da velhice 10,29, os quais podem agravar na pandemia, quando idosos vivenciam luto coletivo, alta letalidade de seu grupo etário, abandono de governantes - por meio de expressões sobre a fragilidade dos contagiados - e falta de políticas públicas de proteção social 30 .

Nesta pesquisa o sentimento de ansiedade, solidão e tristeza durante a pandemia foi mais acentuado entre mulheres idosas do que entre homens idosos. Esses resultados podem ser consequência da carga que a mulher tem no cuidado do ambiente domiciliar, aumentado durante a pandemia, quando muitas idosas são responsáveis pelo cuidado de netos, marido, outros idosos e inclusive de filhos 31 . A maior vulnerabilidade econômica da mulher, decorrente da experiência em seu curso de vida, da desvalorização da sua mão de obra e menor chance de empregos formais 32 , quando comparada aos homens, também pode levar ao maior sentimento de ansiedade em períodos em que há um aumento do desemprego e da pobreza.

Entretanto, autores apontam que deve-se considerar que estereótipos impostos culturalmente 33 levam a diferenças de gênero na experiência emocional e expressividade dos sentimentos ${ }^{34}$, em que mulheres mostram maior expressividade e homens têm experiências emocionais mais fortes como a raiva e a agressividade. Embora seja menor a intensidade do relato de tristeza e solidão na população masculina, seus efeitos podem ser mais fatais, como aponta um estudo de meta-análise sobre a mortalidade por todas as causas 35 .

Identificar e atender, o mais cedo possível, na pandemia, aos idosos que moram sozinhos é uma das recomendações da ONU 3. Estratégias de proximidade e redes de suporte para mitigar a solidão e o distanciamento social incluem o uso de Internet e celular 36 (ICICT/Fiocruz. Sistema de Indicadores de Saúde e Acompanhamento de Políticas Públicas do Idoso (SISAP-Idoso). https://sisapidoso.icict. fiocruz.br/, acessado em 13/Jun/2020). Entretanto, essa opção é limitada em um país como o Brasil, com alto percentual de idosos analfabetos (atingindo mais de $40 \%$ em alguns estados, segundo o último censo) 37 , de famílias pobres que não têm disponibilidade de Internet 38 e com dispositivos móveis não adaptados às limitações do envelhecimento 39 .

Um sistema de saúde que inclua visitas domiciliares foi apontado em um recente relatório da Academia Nacional de Ciências, Engenharia e Medicina dos Estados Unidos como a única conexão eficiente dos idosos com a comunidade 40. Resultados do Estudo Longitudinal de Saúde do Adulto (ELSA) da Inglaterra mostram como estratégias de prevenção primária visando a diminuir o impacto da solidão e do isolamento social podem ajudar a prevenir doenças crônicas entre idosos 26 . No Brasil, os agentes comunitários de saúde (ACS) da Estratégia Saúde da Família têm um papel importante na identificação e acompanhamento de idosos sozinhos e com necessidade de suporte 41. Entretanto, mudanças da Política Nacional de Atenção Básica, desde 2017, debilitaram as equipes dos territórios e desqualificaram o trabalho dos ACS, agravando o risco de desassistência de parte significativa da população 41 , especialmente na pandemia. Teme-se retrocesso quanto ao importante papel da atenção primária na redução de internações e de mortalidade por causas evitáveis em idosos, observado desde o começo do século XXI 42.

A Comissão Econômica para a América Latina e o Caribe (CEPAL), em um estudo recente, comparando o período 2008/2014 com 2014/2018, demonstrou que o Brasil está entre os países com o pior desempenho quanto à pobreza, à desigualdade e ao desemprego, se comparado com outros países da região ${ }^{43}$. O risco de cair na pobreza, pela perda da renda domiciliar, assim como pensões insuficientes para apoiar os padrões de vida, forçam os idosos a continuar trabalhando, inclusive depois de aposentados, ou a fazer arranjos familiares para manter condições de vida minimamente decentes 44 .

A fragilidade da condição laboral e econômica dos idosos brasileiros 45 é evidenciada nesta pesquisa ao identificar alta proporção de idosos que ainda se encontrava trabalhando, sendo grande parte em atividade informal (sem carteira assinada ou trabalhando por conta própria). Além disso, verificou-se diminuição acentuada da renda entre os idosos de pior nível socioeconômico durante a pandemia. 
As mudanças no trabalho dos idosos durante a pandemia impactou homens e mulheres de forma desigual, o que tem relação, provavelmente, com o trabalho feminino no Brasil se concentrar em atividades por conta própria, como diaristas e domésticas, as quais em grande maioria não têm vinculação contratual e estão em situação de maior vulnerabilidade 46.

Os vínculos empregatícios e as garantias trabalhistas são fatores decisivos para garantir as condições de vida seguras e dignas 47 . O crescimento de empregos na informalidade, assim como a flexibilização dos direitos trabalhistas nos anos recentes 48,49 , colocam a população em profunda condição de vulnerabilidade no contexto de deterioração econômica. A perda da renda per capita dos domicílios dos idosos durante a pandemia foi frequente entre os que não tinham vínculo empregatício, o que pode estar relacionado com o aumento da vulnerabilidade econômica da população brasileira desde o ano de 2014, sinalizado pelo estudo da CEPAL 43, e com o aumento do desemprego que o país experimentava durante o primeiro trimestre de 2020, quando houve o primeiro relato da COVID-19 no Brasil 50. Sendo assim, para garantir a sobrevivência dos idosos e assegurar a sustentabilidade e a efetividade das medidas de controle da COVID-19 é preciso instituir políticas de proteção social e apoio a populações em situação de vulnerabilidade, como o programa de renda mínima 51.

Pesquisadores compararam as medidas de controle da epidemia em diferentes países e grau de adesão, e concluíram que as medidas drásticas de distanciamento social, estendidas a toda população, são a opção mais eficiente para a sobrevivência, especialmente dos idosos, apesar da dificuldade desta escolha em muitos contextos sociais 52 . No entanto, essas medidas devem ser acompanhadas de proteção econômica e de interações sociais presenciais ou outras medidas mitigadoras como o apoio domiciliar e comunitário dos serviços de saúde 3 .

Deve-se considerar que o perfil de saúde da população idosa brasileira a torna de alto risco à gravidade da COVID-19, já que a prevalência de doenças crônicas é alta. Com isso, a proteção socioeconômica dos idosos, especialmente com fatores de risco, doenças e comorbidades, permanece imperativa para todos os países 3 . Esta pesquisa mostrou alta prevalência de DCNT associadas ao risco de severidade da COVID-19, como diabetes, hipertensão, doença respiratória crônica, doença do coração ou câncer, resultado que condiz com os riscos analisados com base na Pesquisa Nacional de Saúde (PNS) de 2013 52. Ademais, a falta de diagnóstico oportuno do novo coronavírus 53 representa uma grande dificuldade no Brasil para a proteção e prevenção da gravidade entre os idosos, o que é confirmado pelo baixo porcentual de idosos que fizeram o teste da COVID-19.

Algumas limitações da pesquisa ConVid precisam ser observadas. Destaca-se também que os achados aqui apresentados se referem a uma população que apresenta maior acesso à informação e recursos comunicacionais. Pessoas de menor escolaridade, sem acesso à internet, não puderam participar da pesquisa. A exclusão involuntária das pessoas de baixa escolaridade pode ter provocado a subestimação da proporção de pessoas que não fizeram o distanciamento. Como também, a dependência das observações decorrentes do recrutamento por amostragem em cadeia 54 pode ter levado a estimativas enviesadas. Entretanto, essas limitações foram minimizadas devido ao grande tamanho da amostra e à calibração com os dados da PNAD contínua de 2019, considerando-se a Unidade da Federação de residência, o sexo, a faixa de idade, o grau de escolaridade e a raça/cor como fatores de ponderação.

Outra limitação da pesquisa é não identificar idosos que moram em instituições de longa permanência, modalidade de moradia de milhares de pessoas idosas, com maior risco de contágio e mortalidade por COVID-1955.

A utilização de pergunta sobre ansiedade e depressão sem aplicação de instrumento validado, pode ter levado a superestimar sua prevalência, já que o relato pode referir-se apenas a um sentimento momentâneo e não a uma doença estabelecida. Quanto à renda, as categorias de resposta "mudança significativa" e "ficar sem renda" foram mescladas, o que apresenta uma limitação quanto à avaliação da intensidade da perda. No entanto, foram consideradas graves quaisquer perdas na renda familiar.

Diante disso, pode-se esperar que o quadro de vulnerabilidade seja intensificado em estudos representativos da população idosa. Apesar das limitações expostas, este estudo apresenta apontamentos importantes para o diagnóstico de problemas relacionados às condições de vida de idosos durante a pandemia de COVID-19. Recomenda-se o desenvolvimento de novas pesquisas que sejam representativas da população idosa brasileira e estudos mais aprofundados sobre o impacto da pandemia na saúde desta população. 


\section{Colaboradores}

D. E. Romero participou da concepção, planejamento do estudo, redação, análise e interpretação dos dados, revisão crítica do conteúdo e aprovação da versão final. J. Muzy e G. N. Damacena contribuíram na redação, análise e interpretação dos dados e aprovação da versão final. N. A. Souza, W. S. Almeida, C. L. Szwarcwald, D. C. Mallta, M. B. A. Barros, P. R. B. Souza Júnior, L. O. Azevedo, R. Gracie, M. F. Pina, M. G. Lima, I. E. Machado, C. S. Gomes, A. O. Werneck e D. R. P. Silva colaboraram na análise dos dados, planejamento e revisão crítica do manuscrito.

\section{Informações adicionais}

ORCID: Dalia Elena Romero (0000-0002-26439797); Jéssica Muzy (0000-0003-2526-2317); Giseli Nogueira Damacena (0000-0002-7059-3353); Nathalia Andrade de Souza (0000-0003-13648642); Wanessa da Silva de Almeida (0000-00025164-8603); Celia Landmann Szwarcwald (00000002-7798-2095); Deborah Carvalho Malta (00000002-8214-5734); Marilisa Berti de Azevedo Barros (0000-0003-3974-195X); Paulo Roberto Borges de Souza Júnior (0000- 0002-8142-4790); Luiz Otávio Azevedo (0000-0002-4876-5948); Renata Gracie (0000-0003-0225-3696); Maria de Fátima de Pina (0000-0002-1521-7865); Margareth Guimarães Lima (0000-0001-6996-0745); Ísis Eloah Machado (0000-0002-4678-2074); Crizian Saar Gomes (0000-0001-6586-4561); André Oliveira Werneck (0000-0002-9166-4376); Danilo Rodrigues Pereira da Silva (0000-0003-3995-4795).

\section{Referências}

1. World Health Organization. Director-General's opening remarks at the media briefing on COVID 19. https://www.who.int/dg/ speeches/detail/who-director-general-sopening-remarks-at-the-media-briefing-oncovid-19-11-march-2020 (acessado em 12/ Jun/2020).

2. Huang C, Wang Y, Li X, Ren L, Zhao J, Hu Y, et al. Clinical features of patients infected with 2019 novel coronavirus in Wuhan, China. Lancet 2020; 395:497-506.

3. United Nations. Policy brief: the impact of COVID-19 on older persons. https://www. un.org/development/desa/ageing/wp-con tent/uploads/sites/24/2020/05/COVID-Old er-persons.pdf (acessado em 04/Jun/2020).

4. Rodela T, Tasnim S, Mazumder H, Faizah F, Sultana A, Hossain M. Economic impacts of coronavirus disease (COVID-19) in developing countries. (Working Paper Series). https://osf. io/download/5e88b138d697350021be3353/ (acessado em 12/Jun/2020).

5. Komatsu BK, Menezes-Filho N. Simulações de impactos da COVID-19 e da renda básica emergencial sobre o desemprego, renda, pobreza e desigualdade. São Paulo: Insper Centro de Gestão e Políticas Públicas; 2020. (Policy Paper, 43).

6. Pikhart H, Bobak M, Siegrist J, Pajak A, Rywik S, Kyshegyi J, et al. Psychosocial work characteristics and self rated health in four postcommunist countries. J Epidemiol Community Health 2001; 55:624-30.

7. Yenilmez MI. Economic and social consequences of population aging the dilemmas and opportunities in the twenty-first century. Appl Res Qual Life 2015; 10:735-52.

8. Gragnolati M, Jorgensen $\mathrm{OH}$, Rocha R, Fruttero A. Growing old in an older Brazil: implications of population aging on growth, poverty, public finance and service delivery. Washington DC: World Bank; 2011.

9. United Nations. Political declaration and Madrid international plan of action on ageing, 2002. In: Second World Assembly on Ageing. https://www.un.org/esa/socdev/documents/ ageing/MIPAA/political-declaration-en.pdf (acessado em 13/Jun/2020).

10. Elias N. A solidão dos moribundos: seguido de envelhecer e morrer. Rio de Janeiro: Jorge Zahar Editor; 2001.

11. Szwarcwald CL, Souza Júnior PRB, Damacena GN, Malta DC, Barros MBA, Romero DE, et al. ConVid - Pesquisa de Comportamentos pela Internet durante a pandemia de COVID-19 no Brasil: concepção e metodologia de aplicação. Cad Saúde Pública 2021; 37:e00268320.

12. McKnight $C$, Des Jarlais D, Bramson $H$, Tower L, Abdul-Quader AS, Nemeth C, et al. Respondent-driven sampling in a study of drug users in New York City: notes from the field. J Urban Health 2006; 83 Suppl 6:i54-9. 
13. Szwarcwald CL, Damacena GN. Amostras complexas em inquéritos: planejamento e implicações na análise estatística de dados. Rev Bras Epidemiol 2008; 11 Suppl 1:38-45.

14. Jordan RE, Adab P, Cheng KK. COVID-19: risk factors for severe disease and death. BMJ 2020; 368:m1198.

15. Barros MBA, Lima MG, Malta DC, Szwarcwald CL, Azevedo RCS, Romero D, et al. Relato de tristeza/depressão, nervosismo/ansiedade e problemas de sono na população adulta brasileira durante a pandemia de COVID-19. Epidemiol Serv Saúde 2020; 29:e2020427.

16. Braz MV. A pandemia de COVID-19 (SARSCoV-2) e as contradições do mundo do trabalho. Revista Laborativa 2020; 9:116-30.

17. Afshar S, Roderick PJ, Kowal P, Dimitrov BD, Hill AG. Multimorbidity and the inequalities of global ageing: a cross-sectional study of 28 countries using the World Health Surveys. BMC Public Health 2015; 15:776.

18. Armitage R, Nellums LB. COVID-19 and the consequences of isolating the elderly. Lancet Public Health 2020; 5:e256.

19. Negrini ELD, Nascimento CF, Silva A, Antunes JLF. Elderly persons who live alone in Brazil and their lifestyle. Rev Bras Geriatr Gerontol 2018; 21:523-31.

20. Romero DE. Diferenciais de gênero no impacto do arranjo familiar no status de saúde dos idosos brasileiros. Ciênc Saúde Colet 2002; 7:777-94.

21. Klinenberg E. Social isolation, loneliness, and living alone: identifying the risks for public health. Am J Public Health 2016; 106:786-7.

22. Wu B. Social isolation and loneliness among older adults in the context of COVID-19: a global challenge. Glob Health Res Policy 2020; 5:27.

23. Schmidt B, Crepaldi MA, Bolze SDA, NeivaSilva L, Demenech LM. Saúde mental e intervenções psicológicas diante da pandemia do novo coronavírus (COVID-19). Estud Psicol (Campinas) 2020; 37:e200063.

24. Azeredo ZAS, Afonso MAN. Solidão na perspectiva do idoso. Rev Bras Geriatr Gerontol 2016; 19:313-24.

25. Pantell M, Rehkopf D, Jutte D, Syme SL, Balmes J, Adler N. Social isolation: a predictor of mortality comparable to traditional clinical risk factors. Am J Public Health 2013; 103:2056-62.

26. Valtorta NK, Kanaan M, Gilbody S, Hanratty B. Loneliness, social isolation and risk of cardiovascular disease in the English Longitudinal Study of Ageing. Eur J Prev Cardiol 2018; 25:1387-96

27. Windle K, Francis J, Coomber C. Preventing loneliness and social isolation: interventions and outcomes. London: Social Care Institute for Excellence; 2011.

28. Bozzaro C, Boldt J, Schweda M. Are older people a vulnerable group? Philosophical and bioethical perspectives on ageing and vulnerability. Bioethics 2018; 32:233-9.

29. The Lancet. COVID-19 in Brazil: "So what?". Lancet 2020; 395:1461.
30. Heilborn MLA, Peixoto CE, Barros MML. Tensões familiares em tempos de pandemia e confinamento: cuidadoras familiares. Physis (Rio J.) 2020; 30:e300206.

31. Guiraldelli R. Adeus à divisão sexual do trabalho? Desigualdade de gênero na cadeia produtiva da confecção. Sociedade e Estado 2012; 27:709-32.

32. Plant EA, Hyde JS, Keltner D, Devine PG. The gender stereotyping of emotions. Psychol Women Q 2000; 24:81-92.

33. Deng Y, Chang L, Yang M, Huo M, Zhou R. Gender differences in emotional response: inconsistency between experience and expressivity. PLoS One 2016; 11:e0158666.

34. Rico-Uribe LA, Caballero FF, Martín-María N, Cabello M, Ayuso-Mateos JL, Miret M. Association of loneliness with all-cause mortality: a meta-analysis. PLoS One 2018; 13:e0190033.

35. Newman MG, Zainal NH. The value of maintaining social connections for mental health in older people. Lancet Public Health 2020; 5:e12-3.

36. Käll A, Jägholm S, Hesser H, Andersson F, Mathaldi A, Norkvist BT, et al. Internet-based cognitive behavior therapy for loneliness: a pilot randomized controlled trial. Behav Ther 2020; 51:54-68.

37. Silva TC, Coelho FC, Ehrl P, Tabak BM. Acesso à Internet em períodos recessivos: o caso do Brasil. Revista Ibérica de Sistemas e Tecnologias de Informação 2020; E28:486-98.

38. Câmara TSS, Almeida GKF, Magalhães YC, Almeida WRM. As dificuldades dos idosos com dispositivos móveis. Ceuma Perspectivas 2017; 30:64-75.

39. National Academies of Sciences, Engineering, and Medicine. Social isolation and loneliness in older adults: opportunities for the health care system. Washington DC: National Academies Press; 2020.

40. Sarti TD, Lazarini WS, Fontenelle LF, Almeida APSC. What is the role of primary health care in the COVID-19 pandemic? Epidemiol Serv Saúde 2020; 29:e2020166.

41. Morosini MVGC, Fonseca AF, Lima LD. Política Nacional de Atenção Básica 2017: retrocessos e riscos para o Sistema Único de Saúde. Saúde Debate 2018; 42:11-24.

42. Kanso S, Romero DE, Leite IC, Marques A. A evitabilidade de óbitos entre idosos em São Paulo, Brasil: análise das principais causas de morte. Cad Saúde Pública 2013; 29:735-48.

43. Comissão Econômica para América Latina e o Caribe. Panorama social da América Latina, 2019. Santiago: Organização das Nações Unidas; 2020.

44. D’Alencar RS, Campos JB. Velhice e trabalho: a informalidade como (re)aproveitamento do descartado. Estud Interdiscip Envelhec 2006; 10:29-43.

45. Iriart JA, Oliveira RP, Xavier SS, Costa AM, Araújo GR, Santana VS. Representações do trabalho informal e dos riscos à saúde entre trabalhadoras domésticas e trabalhadores da construção civil. Ciênc Saúde Colet 2008; 13:165-74 
46. Pinto MDV. Regulación del mercado de trabajo y protección social: desafíos institucionales. In: Martínez R, editor. Institucionalidad social en América Latina y el Caribe. Santiago: Comisión Económica para América Latina y el Caribe; 2017. p. 105-66.

47. Krein JD, Colombi APF. A reforma trabalhista em foco: desconstrução da proteção social em tempos de neoliberalismo autoritário. Educ Soc 2019; 40:e0223441.

48. Passos SS, Lupatini M. A contrarreforma trabalhista e a precarização das relações de trabalho no Brasil. Revista Katálysis 2020; 23:13242.

49. Silva MHA, Procópio IM. A fragilidade do sistema de saúde brasileiro e a vulnerabilidade social diante da COVID-19. Rev Bras Promoç Saúde $2020 ; 33: 10724$.

50. Aquino EML, Silveira IH, Pescarini JM, Aquino R, Souza-Filho JA, Rocha AS, et al. Social distancing measures to control the COVID-19 pandemic: potential impacts and challenges in Brazil. Ciênc Saúde Colet 2020; 25 Suppl 1:2423-46

51. Ferguson NM, Laydon D, Nedjati-Gilani G, Imai N, Ainslie K, Baguelin M, et al. Report 9: impact of non-pharmaceutical interventions (NPIs) to reduce COVID-19 mortality and healthcare demand. London: Imperial College London; 2020.
52. Rezende LFM, Thome B, Schveitzer MC, Souza-Júnior PRB, Szwarcwald CL. Adults at high-risk of severe coronavirus disease-2019 (COVID-19) in Brazil. Rev Saúde Pública 2020; 54:50.

53. Gaete R. Análise subnotificação - COVID-19 Brasil. https://ciis.fmrp.usp.br/covid19/ analise-subnotificacao/ (acessado em 07/ Mai/2020).

54. Szwarcwald CL, Souza Júnior PR, Damacena GN, Barbosa Junior A, Kendall C. Analysis of data collected by RDS among sex workers in 10 Brazilian cities, 2009: estimation of the prevalence of HIV, variance, and design effect. J Acquir Immune Defic Syndr 2011; 57 Suppl 3:S129-35.

55. Watanabe HAW, Domingues MARC, Duarte YAO. COVID-19 and homes for the aged: care or an announced death? Geriatr Gerontol Aging 2020; 14:143-5. 


\section{Abstract}

The goal of this study is to characterize the population of older adults in Brazil during the COVID-19 pandemic with regard to health, socioeconomic conditions, gender inequality, adherence to social distancing and feelings of sadness or depression. It is a cross-sectional study carried out with Brazilian older adults who responded to an online health survey $(N=9,173)$, using a "virtual snowball" sampling method. Data were collected online via a self-administered questionnaire. Prevalence and confidence interval estimates were performed and verified for independence using Pearson's chi-square test. During the pandemic there was a fall in household income among almost half of older adults. Extreme social distancing was practiced by 30.9\% (95\% CI: 27.8; $34.1)$ and $12.2 \%(95 \%$ CI: 10.1; 14.7) did not adhere to it. Older adults who were not working before the pandemic adhered in greater numbers to extreme social distancing measures. Most of them presented comorbidities associated with a higher risk of developing the severe form of COVID-19. Feelings of loneliness, distress and sadness were frequent among older adults, especially women. The COVID-19 pandemic widened the inequality gap by affecting the most vulnerable older people. Strategies to mitigate loneliness and social distancing should consider social vulnerability and the marked difference between men and women in terms of household composition and socioeconomic and working conditions. The development of representative surveys of Brazilian older adults is recommended, investigating the impact of the pandemic on this population.

COVID-19; Health of the Elderly; Loneliness; Income; Socioeconomic Factors

\section{Resumen}

El objetivo de este estudio es caracterizar a la población anciana brasileña durante la pandemia de COVID-19, considerando sus condiciones de salud, socioeconómicas, desigualdad de sexo, adhesión al distanciamiento social y sentimiento de tristeza o depresión. Es un estudio transversal realizado con ancianos brasileños que participaron en una encuesta de salud virtual $(N=9.173)$, con un método de muestra "bola de nieve virtual". Los datos fueron recogidos vía web, mediante un cuestionario autocompletado. Se estimaron las prevalencias, intervalos de confianza y, para verificar la independencia de las estimaciones, se utilizó el test chi-cuadrado de Pearson. Durante la pandemia, hubo una disminución de la renta en casi la mitad de los domicilios de los ancianos. El distanciamiento social total fue adoptado por un 30,9\% (IC95\%: 27,8; 34, 1) y 12,2\% (IC95\%: 10,1; 14,7) no se adhirieron. Los ancianos que no trabajaban antes de la pandemia se adhirieron en mayor número a las medidas de distanciamiento social total. Gran parte presentó comorbilidades asociadas a un mayor riesgo de desarrollo de la forma grave de COVID-19. Sentimientos de soledad, ansiedad $y$ tristeza fueron frecuentes entre los ancianos, especialmente entre las mujeres. La pandemia de COVID-19 profundizó la desigualdad al afectar a los ancianos más vulnerables. Se deben elaborar estrategias para mitigar la soledad y el distanciamiento social, teniéndose en cuenta la vulnerabilidad social y la acentuada diferencia entre hombres y mujeres, respecto a la composición domiciliaria $y$ las condiciones socioeconómicas y de trabajo. Se recomienda el desarrollo de investigaciones representativas de la población anciana brasileña, que investiguen el impacto de la pandemia en esta población.

COVID-19; Salud del Anciano; Soledad; Renta; Factores Socioeconómicos
Recebido em 22/Jul/2020

Versão final reapresentada em 16/Dez/2020 Aprovado em 17/Dez/2020 